\title{
Development of site-specific non-intrusive load monitoring for maximum demand control
}

\author{
Azharudin Mukhtaruddin', Fakroul Ridzuan Hashim², Mat Kamil Awang ${ }^{3}$, Husin Mamat ${ }^{4}$, \\ Hafizi Zakaria ${ }^{5}$ \\ ${ }^{1,2,3}$ Faculty of Engineering, Universiti Pertahanan Nasional Malaysia, Malaysia \\ ${ }^{4}$ School of Aerospace Engineering, Universiti Sains Malaysia, Malaysia \\ ${ }^{5}$ Permodalan Nasional Berhad, Malaysia
}

\section{Article Info}

Article history:

Received Oct 23, 2020

Revised Jul 31, 2021

Accepted Aug 19, 2021

\section{Keywords:}

Demand-side load management Edge detection

Electrical energy efficiency

Non-intrusive load management Site-specific

\begin{abstract}
Demand-side load management (DSM) requires greater role-play by endusers. To lower the investment for this load management concept, nonintrusive load management (NILM) was introduced as the solution. However, most of the mathematical techniques used in NILM are complex. This may hinder users from actively take part in the energy management effort. This paper explores the possibilities of applying change point detection techniques with help of differentiation and application of filters. These filters were selected strictly based on site-specific conditions. As part of the NILM implementation, a new and practical technique was developed for this paper. It was found that the developed technique, despite its simplicity it can identify the electrical equipment which added the significant load demand. The performance of the technique was found to be satisfactory as compared to results reported by other researchers.
\end{abstract}

This is an open access article under the CC BY-SA license.

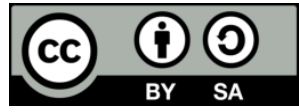

\section{Corresponding Author:}

Azharudin bin Mukhtaruddin

Department of Electrical and Electronic Engineering

Universiti Pertahanan Nasional Malaysia

Kem Sungai Besi, 57000 Kuala Lumpur, Malaysia

Email: azharudin@upnm.edu.my

\section{INTRODUCTION}

Electricity is a vital energy source for our modern society. According to the World Bank, in 2018 almost $89.5 \%$ of the world population has access to electricity while for Malaysia it is 100\% [1]. Malaysia recorded an annual $2.6 \%$ increase in electricity sales and a 3.1\% jumped in maximum demand in 2019 [2]. This is despite the introduction of energy-efficient equipment, programmes and legislation. To reduce the electricity across all sectors, a demand-side management (DSM) unit has been set up under the Malaysian energy commission [3]. DSM concept refers to initiatives and technologies that encourage consumers to optimize their energy use [4]. Among the objectives of DSM are to reduce the peak load or peak load clipping, overall reduction of energy usage and load shifting [5].

Two major approaches for loads monitoring are intrusive and non-intrusive load management (ILM and NILM, respectively) could be considered [6]. The former approach requires the extensive installation of sensors to be installed on every electrical machine. NILM is an interesting approach in which the aggregated data at the power source will be decomposed. From the decomposition data, it is possible to identify the identity and operational status of each machine [7], as if a physical sensor is being installed instead. NILM is also known as the single point measurement approach. Once the machine is identified and its current energy consumption is known, action can be taken on the machine to achieve any or all DSM objectives. 
Even though scores of NILM techniques have been put forward by researchers, it was noted that the main problem is to have it as a reliable solution [8], [9]. An apparent problem with current NILM practice is related to the inaccuracy in the machine's identification and also to the application of complex techniques that eventually hinders users to take part in this technology. This paper is going to promote a method that is ready to be used by most energy players. This will be the main contribution to the NILM study.

Another shortcoming of the current NILM practices is the concentration of the studies mostly on the subject of residential or electrical appliances. This can be proved from the list of data set available for the NILM study [10]. From the list, only about 5 data sets are from sources other than residential or electrical appliances. A lot of other researchers also used data that was measured in a residential building or just measured their data using lab-based electrical consumption setup [11]-[13]. While all the research contributed to the understanding of NILM, more research on the implementation of NILM into business premises is needed [14]. This study echoes the need for real implementation of NILM at a bigger scale. A campus-wide data collection system was selected as the trial ground to the ambition.

\section{RELATED WORKS}

As far as the implementation of NILM on-site is concerned, there are several proposals made available by different researchers. For example, a five-stage implementation of NILM has been proposed by a group of researchers in [15]. Other than that, a three-step general framework of NILM was discussed in [6], [16]. However, authors in [14], [17] proposed a four-step procedure to carry out practical NILM. This proposal was chosen due to its suitability with this study and it is selected as the one followed by this paper. Among the reasons is its flow is logical and it is suitable for site-specific usage. The steps are:

- Data collection and pre-processing.

- Event detection - change of state in the machine's operation over time.

- Feature extraction - unique machine's signature for example in voltage, current and power.

- Load identification.

All steps will be discussed as part of the methodology. For the data collection and pre-processing step, a detailed overview of the system is given as a backdrop on the three scopes or limitations of this study. The limitations are the electrical parameter available for analysis, the duration of the study, and the sampling frequency. All limitations are due to the site condition.

The first limitation is only the electrical current signal was considered in this study. This is the siteconstraint limitation. A lot of discussions have been made on what are the best parameters to be used in NILM. Among the proposals are electrical power, current, and admittance [11], [13], [18]. Nevertheless, the decision to use an electrical current signal is supported by authors in [17], [19], in the former, the authors hold to the opinion that the event is more pronounced in the current waveform.

Another limitation is the period of study. This is again a site-imposed restriction. The final limitation is the data sampling frequency. It was decided that the sampling should be at 5, 10, 15, 30 and 60 minutes. Again, several researchers came out with several proposals on the sampling speed [20], [21]. However, in [22] it was mentioned that a desirable sampling frequency depends on the type of load. Furthermore, not all existing data acquisition systems are readily equipped with high-frequency data sampling capability. This shortcoming gives this research to explore whether alternative sampling time is suitable to be used in NILM. This can also be seen as another contribution of this study.

A group of techniques chosen as the event detection on the aggregate data set is discussed. The event detection as proposed in the landmark paper of NILM may include filtering, differentiating, peak detection [23] and also fitting method [11]. However, for this study, only differentiation and filtering were applied. A detailed explanation of the technique can be found in [8], [11]. While extensive review can be found in [17]. The data sampling frequency is also included in the discussion.

The third step is feature extraction by detecting the edge of the filtered differentiation data. Since the outcome of the filter may be made of sequential data points change point detection technique was applied, in particular edge detection. This technique is used to determine only changes in data points [24]. Finally, for the fourth step, a discussion about the confusion matrix and its application in identifying loads is presented. This common technique is used to show the performance of the proposed techniques. A detailed explanation of the confusion matrix is to be discussed in the methodology section. All analysis using each of the techniques was realised using Simulink ®.

Different from other studies, this study readily has the aggregate and individual data set. Hence the decomposition step was omitted. What this study is going to demonstrate is the application of some relatively common techniques that could also be considered to be used in NILM. Since the techniques employed in this study is not as complex, problem-related NILM explained in the Introduction section can be overcome. On top of that, actual operators of energy management can finally practice NILM as part of their works. 


\section{RESEARCH METHOD}

Data used in this study came from a university campus. The campus has about 20 buildings within 250 acres of land area. Most of the buildings are connected to a central building automation system (BAS). Using the system, a certain level of controls on selected electrical equipment is available. It's also has a very extensive monitoring capability mainly on HVAC, energy monitoring and safety/ security aspects. Monitoring on the main electrical supply for each major building includes parameters such as voltages, currents, power factor, all types of electrical power as well the status of selected circuit breakers. However, at the equipment level, only electrical current is monitored, except for large loads such as chillers that have more monitoring parameters. Hence only current data is considered in this study and it gives the first scope of this study.

The system has a central monitoring system, or workstation (WS), located in a facilities office. From the WS, an operator can monitor a real-time situation of the campus including data on electrical parameters. The data can also be recorded in the WS at a minimum 1-second sampling frequency. The span of the data collection is limited by the available disk space. However, during the visit to evaluate the system, the WS' disk space was low and a lot of electrical monitoring points were unavailable. Therefore, the data collection points (building and machines), the sampling frequency, and the recording span must be carefully selected. Due to this, the second scope of this study is the limitation of the span of data collection to only three days. The recording was started at 0700 on day-1 and ended at 1555 on day-3. However, the analysis will only be done on data started on day- 1 and ended at the end of the business hour on day- 2 .

An administrative building was chosen for the data collection. It has one electricity main switchboard (MSB) which in turn supplies the building. Four pieces of machinery that draw their power from the same MSB were selected. The operation of the building is mainly 0800 to 1700 with a small presence of 24-hour on-duty security personals.

The selection was based on the data available on the machine. They also represent a significant portion of the power delivered by the MSB. One of the machines was a water-cool package air-conditioning unit (WCPU), another was the cooling tower motor (CT) and the other two were water pumps (CDW1 and CDW2). Many of the literature agreed on four-classification of loads [6], [8], [22]. All the above-mentioned loads can be categorised as type II multi-state machines (also known as finite state machines (FSM). It has a repeatable switching pattern. Due to this, it is easier to identify.

The data gathered in this study can be divided into two types: the global data which was sampled at the MSB, and the data taken from four electrical machines. Both types of data were sampled at every 5, 10, 15,30 and 60 minutes, over three days. The sampling frequency is scope number three. The decision of the sampling time was made based on the availability and capability of the system as well as due to a review done in [22] mentioning that a desirable sampling frequency depends on the type of load.

Figure 1 shows an example 5-minute interval of current reading at phase-Y for the MSB, or what is also known as aggregate electrical current data. The current value is represented by the vertical axis while the numbers of data are on the horizontal axis. The latter axis may also be represented with the time of the day. A general pattern can be seen repeated for each day. On the first day, the current reading is around $50 \mathrm{~A}$ before abruptly climbed to about $200 \mathrm{~A}$ within 30 minutes from 0800 hours. It hovers around $250 \mathrm{~A}$ before plunged $150 \mathrm{~A}$ at 1300 and stays there for an hour. It then shoots back to a higher value of about $250 \mathrm{~A}$ at about 1400 up to 1700 . The same pattern repeats for the second day, albeit lower current magnitude and a certain drop at around 1500 due to unknown reasons.

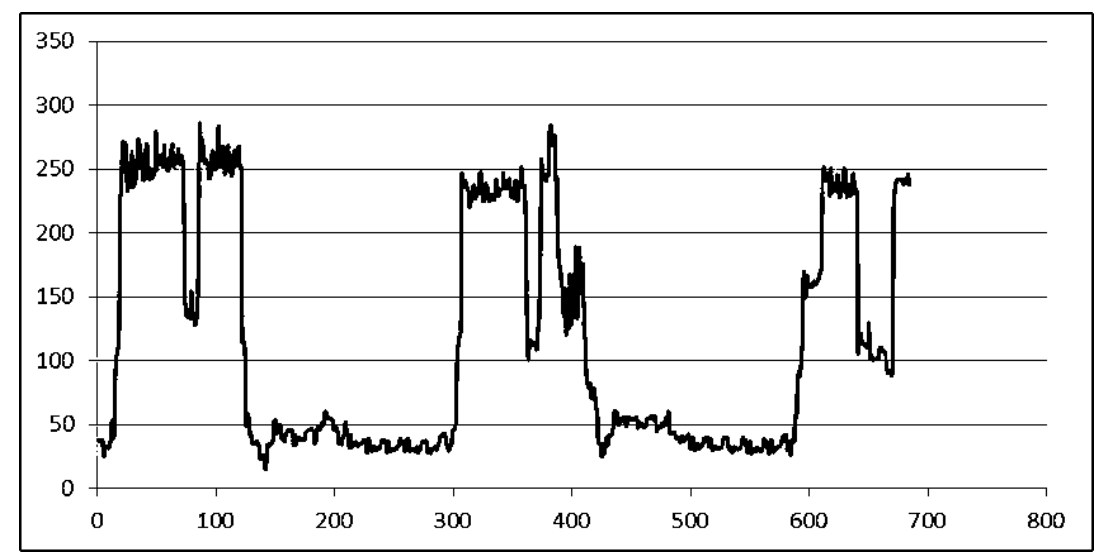

Figure 1. An example of current reading on phase-Y of the MSB 
To process the feature extraction a non-electrical data was employed in this study, specifically the time. The time constraint is between 0800 to 1730 hours. Any data beyond these hours were not considered for the feature extraction. In this work, the output from the event detection was directly used to extract features presumably originated from specific electrical equipment. The data will be going through a series of processes starting with derivation. This first process seeks the magnitude of increase of current from time n, $t_{n}$, to the other, $t_{n+s}$, where $s$ is the sampling frequency. At this stage, detection of the changes in data points is made. Next, the outcome of the derivation is then subjected to a filter. The filter is a high-pass filter, with the magnitude of the filter is selected based on the condition of loads. It will allow a certain value of the derivation to pass. The outcome of the filter is then checked for a positive edge. This step is designed because the output of the filter may span more than one sample point. Subsequently, this edge is used to determine the load identification.

To gauge the performance of the selected methods of disaggregation, [24]-[26] discussed several related indicators. This paper will use precision, recall, and $F_{\beta}$ score as the performance matrices. Terms used in all of the matrices are part of what is called a confusion matrix [25]. Precision for this paper is defined as the ratio of all detected edges (true change point, TP) overall change points, including non-edges, or false change point, FP. Mathematically [24],

$$
\text { precision }=\frac{T p}{\tau p+F p}
$$

Recall, or also known as sensitivity, is the portion of the total TP that is correctly identified. That means this matrix must take into account all edges. This term is known as false non-edges (FN). One way to understand this is to imagine if all edges were detected, FN value must be zero. Hence the recall gives the ratio in which how many TP is detected out of the total actual change point or edge. It is calculated using the following equation [24],

$$
\text { reca } U=\frac{T p}{\tau p+r X}
$$

Notice that precision and recall have different attributes. $\mathrm{F}_{\beta}$ score is given by the following equation [24], with $\beta$ is 1 for a harmonic mean between recall and precision [27]. Hence, both recall and precision are given the same weightage.

$$
F_{\beta} \text { score }=\frac{(1+\beta)^{2} \text { precision xrerail }}{\beta^{2} \text { precision }+ \text { recall }}
$$

\section{RESULTS AND DISCUSSION}

In this section, the data is presented and analyzed according to the four-step implementation discussed in the previous section.

\subsection{Event detection on aggregated electrical current data}

Figure 2 shows the Day-1 data for all aggregated current data. Notice all the positive and negative current changes. All these changes would be subjected to several processes to qualify them as events as discussed in the methodology section. In addition to that, the processes were done for different sampling frequencies. The performances of the processes are assessed using the selected performance matrices.

The subsequent Figure 3 (a) is the derivative of the aggregated current measurement using a 5minute sampling frequency. Because the title of this paper is to cap the maximum demand only positive derivation was going to be inspected. Notice that the highest absolute derivation is about 22.26 A over a 5min period. It is about $10 \%$ of the maximum MSB's current of $229.87 \mathrm{~A}$.

Based on the site condition, filters were selected so that derivatives of only larger than $5 \%, 10 \%$. $15 \%$ and $20 \%$ were allowed to pass. The result is shown in Figure 3 (b) is due to the $10 \%$ filter. The filtered data were then subjected to an edge detection scheme. At this stage, only the incremental edges were detected. Figure 3 (c) is the outcome from the edge detection of Figure 3 (b). Note that the horizontal axis is the point of time. Since the sampling time is 5-minute, the total span will be translated to 0 to 575 minutes. For Figure 3 (a), the vertical axis is the derivation with the unit of A/ 5-minute. On the other hand, the vertical axis for Figure 3 (b) and (c) is the outcome of the edge detection process. It is a binary outcome of either 1 or 0. The output of Figure 3 (b) can be consecutively made of more than one point. 


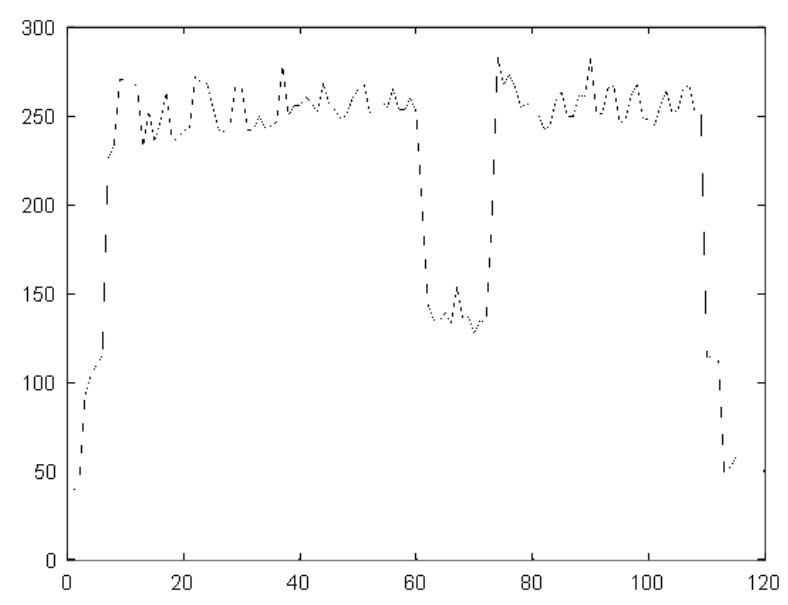

Figure 2. Aggregated current measurement
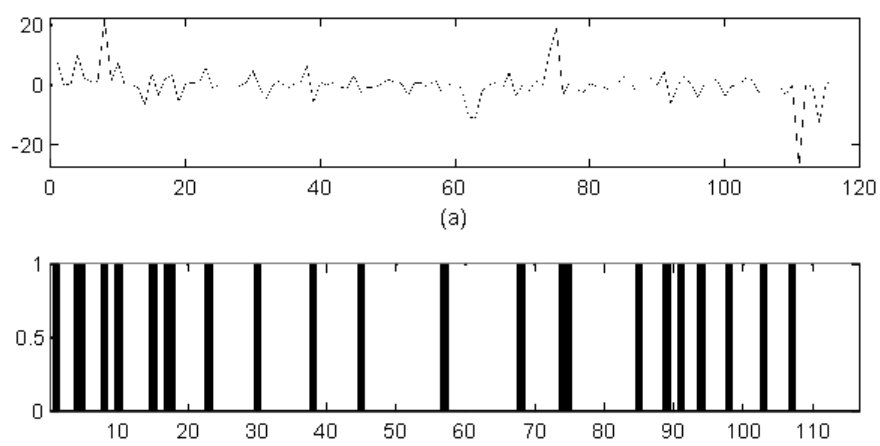

(b)

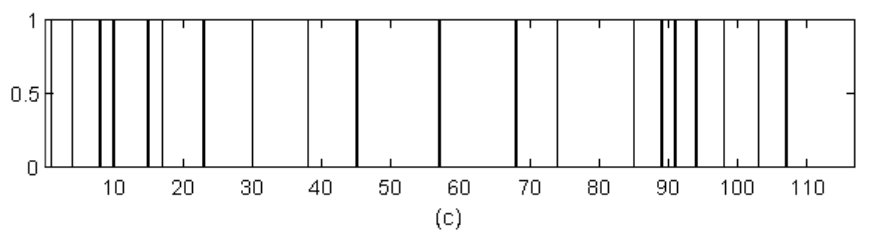

Figure 3. (a) derivation of MSB's current waveform at 5-minute sampling frequency; (b) output of $10 \%$ filter applied to the derivation result; (c) the edge detection output of figure (b)

If the 10-minute sampling frequency is selected and going through all the processes explained previously, the outcome is as shown in Figure 4. From the figure, it can be seen that the information about the aggregated data derivation has now become less. As the result, by applying the same $10 \%$ filter, only two broad passes are registered. Consequently, only two edges are detected as exhibited in Figure 4 (c). This certainly does not reflect the number of significant edges that can be visually scanned from Figure 2 .

With such outcomes, it is safe to conclude that analysis involving data sampled at every 15 -minute and 30-minute will return a worse result. This is largely based on the fact that the derivation for data sampled at both frequencies will yield a smoother outcome. For example, even if the filter is reduced to $5 \%$ for a 10minute sampling frequency, the outcome of the whole process will give a result as shown in Figure 5. Data sampled at 60-minute is not relevant as it exceeded the minimum 30-minute sampling frequency as practised by the utility company.

Figure 6 is the attempt to visualize the edge detection results with respect to the original MSB's current. Figure 6 (a)-(d) is the edge detection result on filter 5\%,10\% 15\%, and $20 \%$ respectively. Visually it can be concluded that the smaller the filter, the more signals were passed for the edge detection process is shown in Figure 6. However, no accurate performance of the filtering and edge detection process could be concluded. 


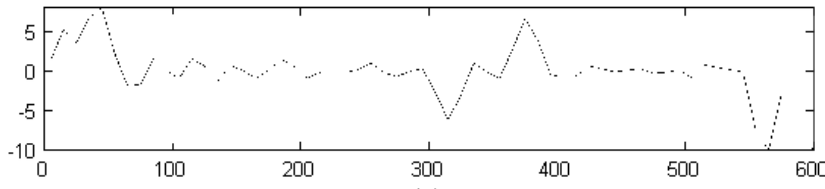

(a)

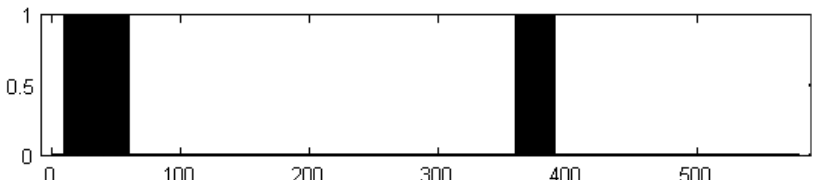

(b)

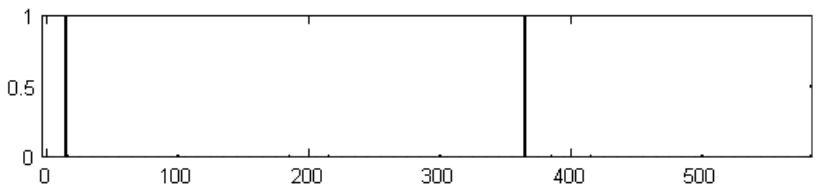

(c)

Figure 4. (a) Derivation of MSB's current waveform at 10-minute sampling frequency; (b) output of $10 \%$ filter applied to the derivation result; (c) the edge detection output of figure (b)

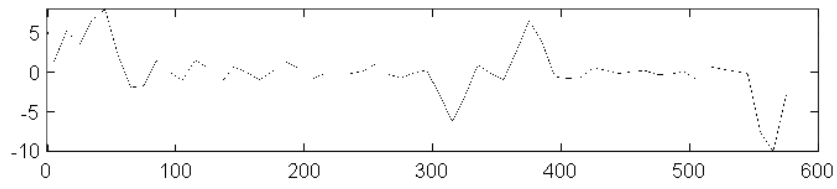

(a)

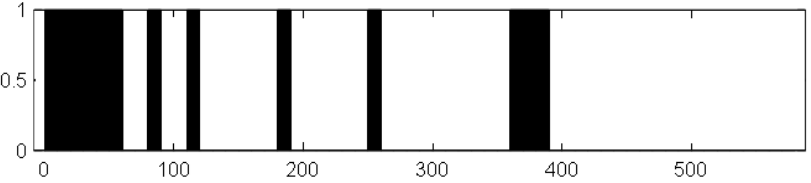

(b)

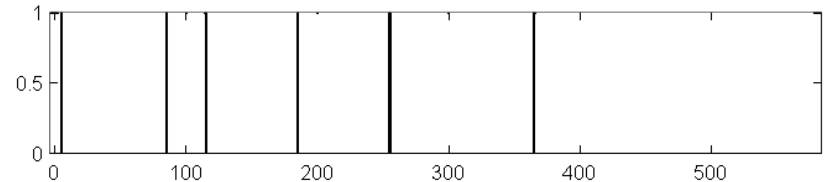

(c)

Figure 5. (a) Derivation of MSB's current waveform at 10-minute sampling frequency; (b) output of 5\% filter applied to the derivation result; (c) the edge detection output of figure (b)

Data in Table 1 on the other hand provide the achievement of performance. Note that for filters at $10 \%$ and $15 \%$, the recall, precision and F1 score all achieve the mark of 1 . This means all possible significant changes for the $10 \%$ and $15 \%$ filters were correctly detected by the edge detection process. Therefore, both filters are the best in this event detection stage. Nevertheless, for the 5\% filter, the recall score is 0.5625 points, while the precision is good at 0.8182 points. For the $20 \%$ filter, the precision is registered only at 0.4783 points, even though the recall matrix has a perfect 1 point. Hence, although the $20 \%$ filter is capable of correctly identified TP from all events, it is far from capturing all available change points.

Table 1. Performance index of edge detection on MSB's current waveform

\begin{tabular}{ccccc}
\hline Filter & $5 \%$ & $10 \%$ & $15 \%$ & $20 \%$ \\
\hline Recall & 0.5625 & 1.0000 & 1.0000 & 1.0000 \\
Precision & 0.8182 & 1.0000 & 1.0000 & 0.4783 \\
F1 score & 0.6667 & 1.0000 & 1.0000 & 0.6471 \\
\hline
\end{tabular}

Development of site-specific non-intrusive load monitoring for maximum... (Azharudin Mukhtaruddin) 


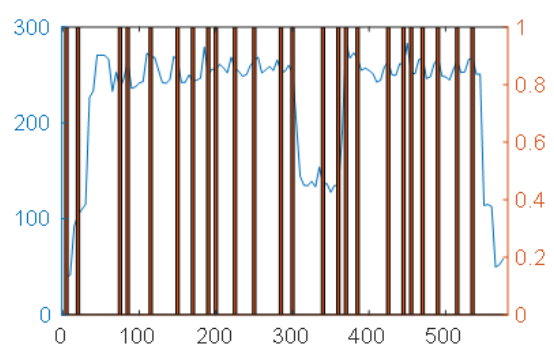

(a)

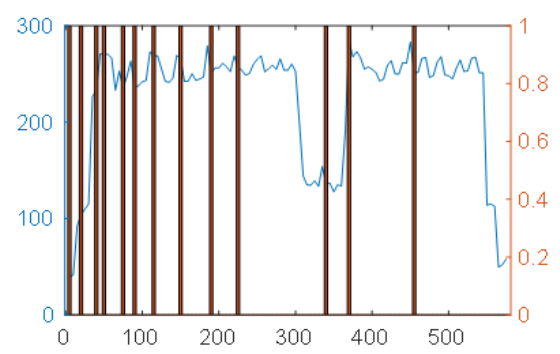

(c)

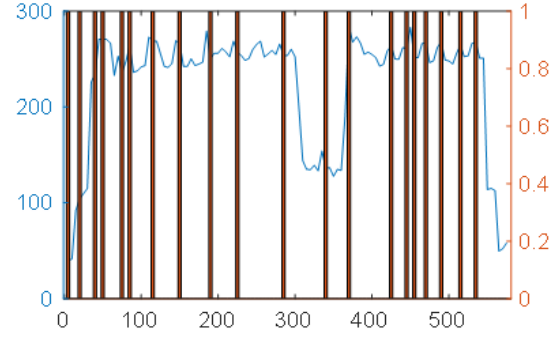

(b)

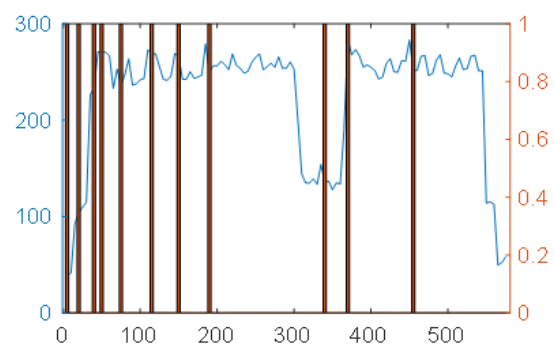

(d)

Figure 6. Visual interpretation of edge detection outcome as imposed on the original aggregated current data

\subsection{Feature extraction and load identification}

The individual selected electrical load data is as shown in Figure 7. Notice that the WCPU load is only operational within the office hour with a temporary shutdown of about an hour at noon. Appropriately, the CT and CDW1 and CDW2 are also expected to be operational in the same period. However, the actual operating time for both is 30-minute before and after the normal working hour. The way WCPU operating is to run at a base current demand between $20.1 \mathrm{~A}$ to $21 \mathrm{~A}$. It fluctuates in the range of $33.4 \mathrm{~A}$ to $35.9 \mathrm{~A}$. These fluctuations are due to the cut-in and cut-off operation of an additional compressor to maintain the room's set temperature.

The CT and CDWs operation are closely related to each other. From Figure 9, it can be seen that the starting and shutting downtime of CT and CDWs are identical. However, due to the alternating policy between CDW1 and CDW2 operation was in place, CDW1 would operating in the first half of the office hour while CDW2 for the other half. It is important to remark that the fluctuations in CT and CDWs data sets are found to be loosely correlated. Statistically, the analysis showed that the R2 value between both data is small. On top of that, the standard deviation for CT is $0.2 \mathrm{~A}$, for CDW1 is $0.56 \mathrm{~A}$, and for CDW2 is $0.31 \mathrm{~A}$. It is also can be noticed that the operational time is related to WCPU. This is expected as the function of CT and CDWs are to cool down the compressors in the WCPU. As with the CT-CDWs fluctuations relationship, the correlations between are very low.

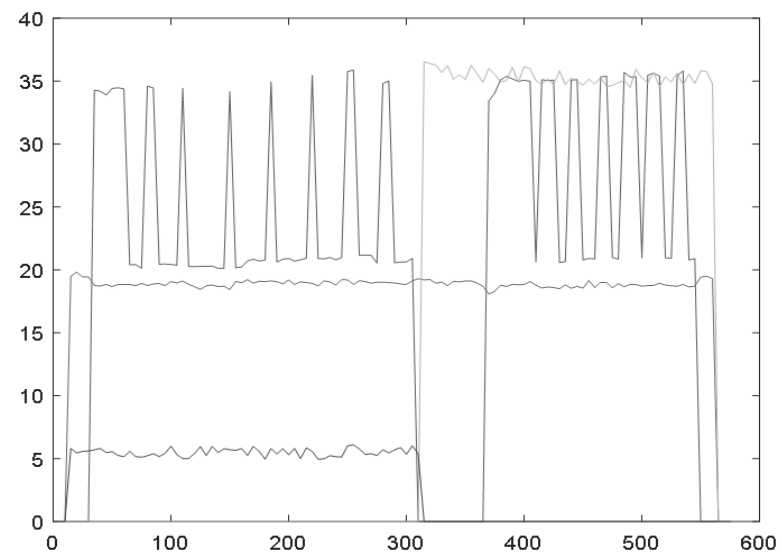

Figure 7. Current curves for WCPU, CT, CDW1 and CDW2 
Starting of CT and CDW1 was registered as significant derivative value and subsequently detected as incremental edge but beyond the working hour. Even for CDW2, the off position at $\mathrm{t}=315$-minute has only contributed to the negative derivation value in the total MSB current. All CT and CDWs have been classified as type II electrical machines. However, since all CT, CDW1 and CDW2 cannot be shut down as long as any WCPU is operational, may cause them to also be classified as type IV.

Notice that the CDW2 started at $\mathrm{t}=315$-minute. However, this change was not picked up by the edge detection because the derivation value on the total MSB current is negative. The sudden increment will probably never be registered as a positive edge on the aggregated current waveform. This is because the policy says most WCPUs will be switched off during the break hour starting at $t=315$-minute. Hence CDW2 would never be important to be positively identified.

Interestingly, the base current demand is about the same as the highest derivation magnitude of the aggregated current data. At this stage, it is logical to assume that the fluctuations at the aggregated current curve can be traced its origin to WCPU's demand. In other words, the features of WCPU's current curve can be revealed from the event detection done at the MSB's current waveform.

Figure 8 shows, (a) the derivation of WCPU's current curve, (b) the output of $10 \%$ filter, and (c) the edge detection of (b). All processes were done on the 5-minute sampling data. Again, the vertical axis for Figure 8 (a) is the derivation value, A/ 5-minute. All horizontal axes are in time (minute). Notice that the derivation outcomes are almost pulse-shaped. Hence, all the outputs of the filter are pulses. The edge detection is naturally pulse-type output. Therefore, the outcomes of edge detection are identical to those found in Figure 8 (b).

The edge detection process on the WCPU's current data yielded the outcomes as shown in Figure 9. Visually it can be generally deduced that outcomes of edge detection due to 5\%, $10 \%$ and $15 \%$ filters, Figure 9 (a)-(c) respectively, are quite similar. The worse outcome is clearly can be seen in Figure 9 (d). It is the result of edge detection for a $20 \%$ filter. Only two edges of the current curve can be detected.

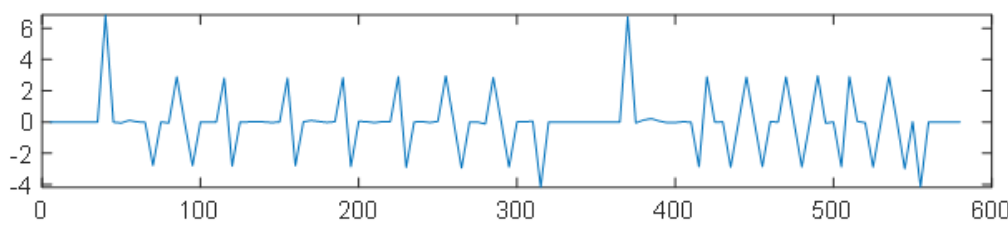

(a)

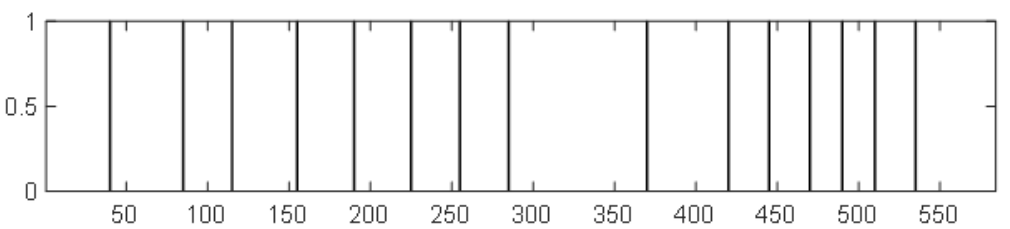

(b)

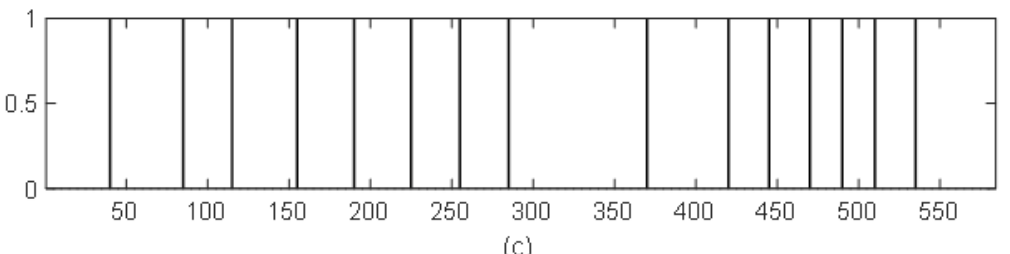

Figure 8. (a) Derivation of WCPU's current waveform at the 5-minute sampling frequency;

(b) output of $10 \%$ filter applied to the derivation result; (c) the edge detection output of figure (b)

As expected, recall and precision matrices for 5\%,10\% and $15 \%$ filters all return 1.0000 points as shown in Table 2. It means the process is able to capture all edges and perfectly distinguished between positive edges and non-edges. The following table is the tabulation of the results. For the $20 \%$ filter, it is capable to capture all edges it managed to detect, which are only two. This is reflected by the very poor performance in precision. 
Table 2. Performance index of edge detection on WCPU's current waveform

\begin{tabular}{ccccc}
\hline Filter & $5 \%$ & $10 \%$ & $15 \%$ & $20 \%$ \\
\hline Recall & 1.0000 & 1.0000 & 1.0000 & 1.0000 \\
Precision & 1.0000 & 1.0000 & 1.0000 & 0.1000 \\
F1 score & 1.0000 & 1.0000 & 1.0000 & 0.1818 \\
\hline
\end{tabular}

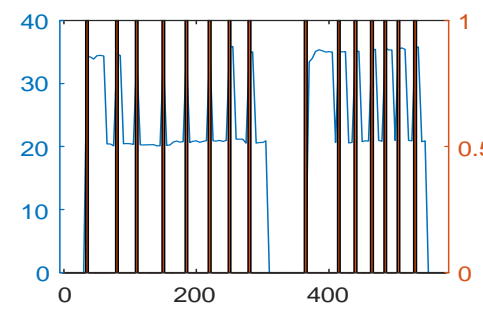

(a)

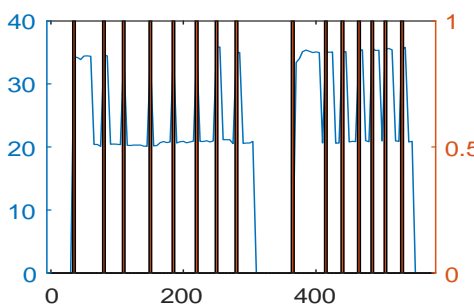

(c)

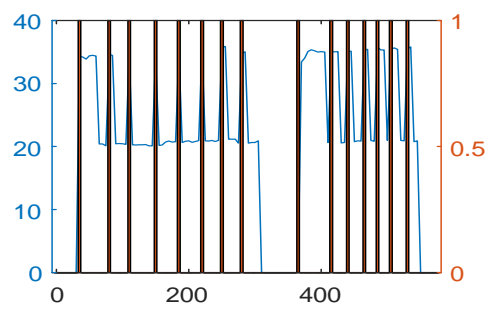

(b)

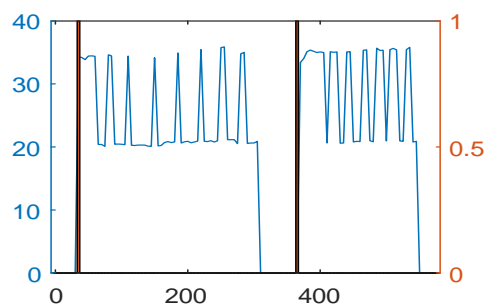

(d)

Figure 9. Current curves for WCPU, CT, CDW1 and CDW2

With a correct selection of filter selection, changes in WCPU's operation can be confidently detected. If those changes are significantly reflected in the aggregated current data, it can be perceived as the feature extracted from the equipment. In the case of this study, the equipment itself can be directly identified.

Table 3 is the performance indexes of the change point in MSB's current curve with a change point in WCPU's current curve as its predictor. The best performance is when the $10 \%$ filter was used to analyze MSB's data against the outcome of edge detection on WCPU's data using 5\%, 10\% and 15\% filters.

Table 3. Performance index between edges in aggregated current data and edges in WCPU current data

\begin{tabular}{ccccc}
\hline Filter & $5 \%$ & $10 \%$ & $15 \%$ & $20 \%$ \\
\hline Recall & 0.6667 & 0.7333 & 0.3333 & 0.2667 \\
Precision & 0.4000 & 0.5500 & 0.3846 & 0.3636 \\
F1 score & 0.5000 & 0.6286 & 0.3571 & 0.3077 \\
\hline
\end{tabular}

It can be seen that the result in Table 3 and in [12] are comparable, looking at the average values. The result from [12], which was based on the domestic data, is as shown in Table 4. Furthermore, it was reported in the same paper that another study by Salerno (2018) yielded an average F1 score of 0.67. Table 5 is the result as reported in [28]. Comparing the result from this study and [28] also shows a good agreement.

Table 4. Performance index [12]

\begin{tabular}{cccccc}
\hline Apparatus & Kettle & Washing machine & Dish washer & Fridge & Average \\
\hline Recall & 0.47 & 0.40 & 0.90 & 0.96 & 0.68 \\
Precision & 0.58 & 0.43 & 0.73 & 0.97 & 0.68 \\
F1 score & 0.52 & 0.41 & 0.81 & 0.97 & 0.68 \\
\hline
\end{tabular}

Table 5. NILM performance [28]

\begin{tabular}{cccc}
\hline Current value & $50 \mathrm{~Hz}$ & 50 and $150 \mathrm{~Hz}$ & $50,150,250 \mathrm{~Hz}$ \\
\hline Recall & 0.536 & 0.635 & 0.624 \\
Precision & 0.528 & 0.651 & 0.639 \\
F1 score & 0.566 & 0.659 & 0.643 \\
\hline
\end{tabular}




\section{CONCLUSION}

Clearly that using the common techniques proposed in this paper, significant energy-consuming equipment can be revealed just by analysing the aggregated current demand. The performance of the technique is comparable to other research. This technique is site-specific and it is justifiable as different sites may have different electrical load settings, different energy management policies and even have specific limitations, not unlike the site undertaken under this study. Further study on a more complex system is needed in order to verify the method used in this paper. Of course, the disaggregation process needs to be included too. A longer period of study is also preferable to develop a more robust method. Nevertheless, the method is based on a simple mathematical principle. It is then applied to a very specific site condition. The lacking of complexity in this technique itself can attract the operators of the energy efficiency industry. Furthermore, as the actual persons in handling the matter, the operators are all well-versed with their system and know the limitation of the system in order to fine-tune its reliability and robustness. Practically speaking, for this site, it is possible to explain the increase in the global electrical data is due to WCPU at a $10 \%$ filter. From this possibility, the operator can make an objective decision to alter the WCPU's operation in order to achieve desirable maximum demand control.

\section{ACKNOWLEDGEMENTS}

This study was made possible with the Fundamental Research Grant Scheme (FRGS/1/2020/TK0/UPNM/03/3) made available by the Ministry of Higher Education, Malaysia. The authors would like to express their appreciation to Development Department, Engineering Campus, Universiti Sains Malaysia, Penang, Malaysia, for permission for accessing their system and to use the data for this study.

\section{REFERENCES}

[1] World Bank, "Access to electricity (\% of the population)." https://data.worldbank.org/indicator/EG.ELC.ACCS.ZS?end=2018\&start=1990\&view=chart (accessed May 01, 2020).

[2] Malaysia Energy Commission, Peninsular Malaysia Electricity Supply Industry Outlook, 2019. Accessed: May 2020. [Online]. Available: https://www.st.gov.my/en/contents/files/download/106/Peninsular_Malaysia_Electricity_Supply_Industry_Outlook _2019_compressed.pdf

[3] Malaysia Energy Commission, Energy Malaysia: Strengthening the Future of Energy in Malaysia. Accessd: May 01, 2020. [Online]. Available: https://www.st.gov.my/en/contents/files/download/112/Energy_Malaysia, Volume_14,_20185.pdf

[4] Energy Market Authority of Singapore, "Demand Side Management," Overview of Electricity Market. https://www.ema.gov.sg/Demand_Side_Management.aspx (accessed May 01, 2020).

[5] E. Sarker et al., "Progress on the Demand Side Management in Smart Grid and Optimization Approaches," International Journal of Energy Research, vol. 45, no. 1, pp. 36-64, Jan. 2021, doi: 10.1002/er.5631.

[6] O. Hamid, M. Barbarosou, P. Papageorgas, K. Prekas, and C. T. Salame, "Automatic Recognition of Electrical Loads Analyzing the Characteristics Parameters of the Consumed Electric Power Through a Non-Intrusive Methodology," in Energy Procedia, 2017, pp. 742-752, doi: 10.1016/j.egypro.2017.07.137.

[7] Abubakar, S. N. Khalid, M. W. Mustafa, H. Shareef, and M. Mustapha, "Application of load monitoring in appliances energy management - A review," Renewable and Sustainable Energy Reviews, vol. 67, pp. 235-245, 2017, doi: 10.1016/j.rser.2016.09.064.

[8] M. Hamdi, H. Messaoud, and N. Bouguila, "A New Approach of Electrical Appliance Identification in Residential Buildings," Electric Power Systems Research, vol. 178, no. September 2019, p. 106037, 2019, doi: 10.1016/j.epsr.2019.106037.

[9] C. Lam, Z. Zheng, and X. Luo, "Electricity Load Decomposition Prototype for Household Appliances: System Design and Development," in Energy Procedia, 2019, vol. 158, pp. 3158-3163. doi: 10.1016/j.egypro.2019.01.1018.

[10] NILM datasets. Accessed Jan. 30, 2021 [Online]. Available: https://github.com/nilmtk/nilmtk/search?q=NILM+datasets

[11] Y. Xiao, Y. Hu, H. He, D. Zhou, Y. Zhao, and W. Hu, "Non-Intrusive Load Identification Method Based on Improved KM Algorithm," IEEE Access, vol. 7, pp. 151368-151377, 2019, doi: 10.1109/ACCESS.2019.2948079.

[12] C. Puente, R. Palacios, Y. González-Arechavala, and E. F. Sánchez-Úbeda, "Non-Intrusive Load Monitoring (NILM) for Energy Disaggregation Using Soft Computing Techniques," Energies, vol. 13, no. 12, p. 3117, 2020, doi: $10.3390 /$ en 13123117.

[13] S. Welikala, S. Member, C. Dinesh, and S. Member, "Incorporating Appliance Usage Patterns for Non-Intrusive Load Monitoring and Load Forecasting," IEEE Transactions on Smart Grid, vol. 10, no. 1, pp. 448-461, 2019, doi: 10.1109/TSG.2017.2743760. 
[14] M. Zhuang, M. Shahidehpour, and Z. Li, “An overview of Non-intrusive: Approaches, Business Applications and Challenges," in 2018 International Conference on Power System Technology, 2018, pp. 4291-4299, doi: 10.1109/CENCON.2015.7409513.

[15] T. Kien, E. Dekneuvel, G. Jacquemod, B. Nicolle, and O. Zammit, "Electrical Power and Energy Systems Development of a Real-Time Non-Intrusive Appliance Load Monitoring System : An Application-Level Model," International Journal of Electrical Power and Energy Systems, vol. 90, pp. 168-180, 2017, doi: 10.1016/j.ijepes.2017.01.012.

[16] C. Nalmpantis and D. Vrakas, "Machine Learning Approaches for Non-Intrusive Load Monitoring: From Qualitative to Quantitative Comparison," Artificial Intelligence Review, vol. 52, pp. 217-243, 2018, doi: 10.1007/s10462-0189613-7.

[17] Ruano, A. Hernandez, J. Ureña, M. Ruano, and J. Garcia, "NILM Techniques for Intelligent Home Energy Management and Ambient Assisted Living: A Review," Energies, vol. 12, no. 2203, pp. 1-29, 2019, doi: 10.3390/en12112203.

[18] Y. Liu, X. Wang, L. Zhao, and Y. Liu, "Admittance-Based Load Signature Construction for Non-Intrusive Appliance Load Monitoring," Energy \& Buildings, vol. 171, pp. 209-219, 2018, doi: 10.1016/j.enbuild.2018.04.049.

[19] S. Ghosh, A. Chatterjee, and D. Chatterjee, "Improved Non-Intrusive Identification Technique of Electrical Appliances for a Smart Residential System," IET Generation, Transmission and Distribution, vol. 13, no. 5, pp. 695-702, Mar. 2019, doi: 10.1049/iet-gtd.2018.5475.

[20] Alkhulaifi and A. J. Aljohani, "Investigation of Deep Learning-based Techniques for Load Disaggregation, LowFrequency Approach,” 2020. [Online]. Available: www.ijacsa.thesai.org

[21] Klemenjak and S. Makonin, "Towards Comparability in Non-Intrusive Load Monitoring: On Data and Performance Evaluation," in 2020 IEEE Power \& Energy Society Innovative Smart Grid Technologies Conference (ISGT), 2020, doi: 10.1109/ISGT45199.2020.9087706.

[22] N. Farahin, P. Abdullah, and M. Yusri, "A Review Disaggregation Method in Non-Intrusive Appliance Load Monitoring," Renewable and Sustainable Energy Reviews, vol. 66, pp. 163-173, 2016, doi: 10.1016/j.rser.2016.07.009.

[23] G. W. Hart, "Nonintrusive Appliance Load Monitoring," Proceedings of the IEEE, vol. 80, no. 12, pp. 1870-1891, 1992, doi: 10.1109/5.192069.

[24] S. Aminikhanghahi and D. J. Cook, "A Survey of Methods for Time Series Change Point Detection," Knowledge And Information Systems, vol. 51, no. 2, pp. 339-367, 2017, doi: 10.1007/s10115-016-0987-z.

[25] N. Tatbul, T. J. Lee, S. Zdonik, M. Alam, and J. Gottschlich, "Precision and Recall for Time Series," in Advances in Neural Information Processing Systems, 2018, pp. 1920-1930.

[26] N. Singh and C. Olinsky, "Demystifying Numenta Anomaly Benchmark," in 2017 International Joint Conference on Neural Networks (IJCNN), 2017, pp. 1570-1577, doi: 10.1109/IJCNN.2017.7966038.

[27] C. Truong, L. Oudre, and N. Vayatis, "Selective Review of Offline Change Point Detection Methods," Signal Processing, vol. 167, p. 107299, 2020, doi: 10.1016/j.sigpro.2019.107299.

[28] E. P. Loukas et al., "A Machine Learning Approach for NILM based on Odd Harmonic Current Vectors," Proceedings of 2019 8th International Conference on Modern Power Systems, MPS 2019, pp. 1-6, 2019, doi: 10.1109/MPS.2019.8759666. 